\title{
Factores Ambientales y Psicosociales Vinculados a Síntomas de Ataque de Pánico Después del Terremoto y Tsunami del 27 de Febrero de 2010 en la Zona Central de Chile
}

\section{Environmental and Psychosocial Factors Associated with Panic Attack Symptoms After the February 272010 Earthquake And Tsunami in the Central Zone of Chile.}

\author{
Marcelo C. Leiva \\ Gonzalo R. Quintana \\ Universidad de Talca, Chile. \\ (Rec: 5 de agosto de 2010 / Acep: 21 de octubre de 2010)
}

\begin{abstract}
Resumen
La presente investigación proporciona una primera evaluación de síntomas de ataque de pánico y su relación con algunas variables asociadas con la magnitud del daño sufrido por las personas expuestas al terremoto del 27 de Febrero de 2010 en la zona central de Chile.

Un total de 150 habitantes de diversas localidades fueron evaluados para determinar la presencia de síntomas de ataque de pánico dentro de las dos semanas posteriores a la catástrofe. Los resultados indican más síntomas de crisis de pánico en aquellas personas que sufrieron pérdidas de bienes y en aquellos que estuvieron expuestos al tsunami o riesgo de tsunami.

Se discute la necesidad de evaluar otros trastornos (p.e., estrés post-traumático) y poblaciones (p.e., niños), así como también la importancia de crear indicadores cuantitativos del impacto de estas catástrofes, basados en la combinación de variables tales como la intensidad del sismo y la magnitud del daño personal.

Palabras clave: Terremoto, tsunami, ataque de pánico, 27 de febrero de 2010.
\end{abstract}

\begin{abstract}
The present research provides a first screening of the presence of panic attack symptoms and their relation to some variables associated with the magnitude of the damage suffered by individuals exposed to the earthquake occurred on February 272010 in the central zone of Chile.

A total of 150 adults that lived in several cities and villages of the central zone of Chile were assessed to determine the presence of panic attack symptoms within the first two weeks after the catastrophe. The results indicate more physical and psychical panic attack symptoms on those individuals that lose some of their belongings and on those that were exposed to the tsunami or risk of tsunami. The discussion emphasizes the need for evaluating further disorders (e.g., post-traumatic stress) and to examine at-risk populations (e.g., children). It is suggested the importance of creating quantitative indexes based on variables such as extent of the loss, physical damage and quake intensity, to asses the individual impact of this sort of catastrophes.
\end{abstract}

Key words: Earthquake, tsunami, panic attack, february 272010.

Correspondencia sobre el artículo enviar a: Marcelo C. Leiva, Facultad de Psicología, Universidad de Talca, Talca, Chile. Casilla No 747 - Talca Dirección: Av. Lircay s/n Talca. Teléfono: +56 (71) 201654; Fax: +56 (71) 201510. E- mail: mleiva@utalca.cl. El presente estudio está enmarcado en el Programa de Investigación "Calidad de Vida y Ambientes Saludables" de la Facultad de Psicología, y financiado por la Dirección de Programas de Investigación de la Universidad de Talca, Chile. 


\section{Introducción.}

El sábado 27 de febrero a las 03:34 hora continental chilena, se produjo un terremoto de casi 3 minutos de duración que alcanzó una magnitud de 8,8 en la escala de Magnitud Local (Richter). Su epicentro se ubicó frente a las localidades de Curanipe y Cobquecura, cerca de 150 kilómetros al noroeste de Concepción y a 63 kilómetros al suroeste de Cauquenes (USGS, 2010).

Debido, entre otros factores, a la localización submarina del epicentro y su proximidad con la costa, dicho movimiento telúrico produjo un tsunami que afectó las costas del valle central entre las regiones del Libertador Bernardo O’Higgins hasta la del Bío-Bío. En Constitución (ciudad costera de la región del Maule), media hora después del terremoto, tres olas de 8,10 y 8 metros respectivamente, devastaron la ciudad. En localidades cercanas como Pichilemu, Pelluhue, Curanipe, Iloca y Coi Coi, el mar penetró hasta 200 metros al interior. En el puerto de Talcahuano (región del Bío-Bío), olas de hasta 5 metros destruyeron prácticamente toda la zona pesquera, uno de los principales sustentos de la ciudad (Riquelme, P., 2010).

Dada la ubicación e intensidad de ambos fenómenos (el quinto terremoto más intenso registrado; USGS, 2010), esta catástrofe natural marca un antes y un después, configurándose como una de las más importantes en la historia nacional e internacional. Las regiones más afectadas por el terremoto fueron: Valparaíso, Metropolitana, O’Higgins, Maule, Bío-Bío y La Araucanía, donde viven alrededor de 12 millones de personas, un $80 \%$ de la población total del país (INE, 2003). Las principales ciudades de las regiones del Maule y del Bío-Bío sufrieron graves daños en su patrimonio arquitectónico. Las del interior, por el terremoto (Curicó, Talca y Concepción) y las costeras, por el terremoto y el tsunami (Constitución y Talcahuano). En total, unas 500 mil viviendas presentan daños severos que dejan cerca de 2 millones de personas damnificadas (Riquelme, C., 2010). Las víctimas fatales fueron 521 personas, con un saldo de 56 que aún continúan desaparecidas (Ministerio del Interior, 2010a; 2010b).

$\mathrm{Al}$ igual que en otros eventos traumáticos (p. e., guerra, secuestros, huracanes), las personas frente a este tipo de fenómenos naturales son expuestas a situaciones que vulneran sus capacidades, alterando su salud mental tanto a corto como a largo plazo (Rochanakorn, 2007). La Organización Panamericana de Salud (OPS, 2006) plantea que el impacto de cualquier evento traumático depende de tres factores: la naturaleza del evento (tipo de evento, duración, intensidad, sorpresa y cantidad de personas afectadas), las características personales y la vulnerabilidad de las víctimas (mediadas por variables tales como el sexo, edad y nivel socio-económico) y el entorno o circunstancias en donde se produce (contexto social, cultural e histórico del evento). De este modo, a casi 6 meses del evento, es evidente el impacto y las innumerables consecuencias que este desastre provocó, tales como la pérdida de seres queridos, la destrucción de su ambiente inmediato, la destrucción parcial o total de casas y posesiones materiales y el desempleo, por mencionar las más importantes.

A esto se suman las consecuencias en la salud mental provocadas tanto por la catástrofe en sí como por todos sus efectos colaterales. Según la OPS, los trastornos más frecuentes son los de ansiedad, dentro de los cuales se encuentra el trastorno por estrés post-traumático (TEPT) y la crisis de angustia o ataque de pánico (CAPA); los trastornos del ánimo como la depresión; los trastornos orgánicos de causa psíquica; el consumo excesivo de alcohol y drogas; y las conductas violentas, como la violencia intrafamiliar (OPS, 2006; Solvason, Ernst y Roth, 2003). Algunos de estos desórdenes mejoran con el paso del tiempo, mientras que otros se mantienen incluso hasta años después de la catástrofe (McFarlane y Papay, 1992).

La prevalencia de este tipo de trastornos es una constante en todos los estudios realizados después de una catástrofe. En el caso del TEPT, su prevalencia en una población normal no supera el 14\% (López-Ibor y Valdés, 2008). En contraste con esto, después del terremoto ocurrido en 1993 en Marathwada, India, un $23 \%$ de la población presentó TEPT (Sharan, Chaudhary, Kavathekar y Saxena, 1996), mientras que en el terremoto ocurrido el año 2007 en Pisco, Perú, la prevalencia fue de 25\% (Cairo, Dutta y Nawaz, 2010). La misma tendencia se repite en grupos considerados de alto riesgo. Por ejemplo, un predominio de síntomas de TEPT y ansiedad han sido encontrados en niños que estaban más cerca del epicentro del terremoto del año 1999 ocurrido en Grecia (Groome y Soureti, 2004), en mujeres presentes en el terremoto del año 2005 en Pakistán (Khan, Masood, Mukhtar, Sana y Chaudhry, 2010), en ancianos luego del terremoto del 1989 en Australia (Ticehurst, Webster, Carr y Lewin, 1996) y en personas pertenecientes a niveles socioeconómicos bajos (Kar, Mohapatra, Nayak, Pattanaik, Swain y Kar, 2007). Por otro lado, los síntomas vinculados con trastornos como la depresión, ansiedad y CAPA son otros de los más frecuentes en este tipo de fenómenos, repitiéndose la misma tendencia que en el TEPT en niños (Thienkrua, et al., 2006), adolescentes (Kar, et al., 2007) y mujeres (Harville, Xiong, Buekens, Elkind-Hirsch y Pridjian, 2009).

Cabe señalar que estos síntomas no siempre se presentan inmediatamente después de una catástrofe. De hecho, en el caso del TEPT, los síntomas pueden aparecer incluso 6 meses después o más (Priebe, Grappasonni, Mari, Dewey, Petrelli y Costa, 2009). No obstante, según lo señalado por Cohen (2008), es preciso tener en cuenta que la presencia de este tipo de síntomas no implica necesariamente la existencia de una patología, y que al observar y documentar síntomas después de un desastre, estos deberían interpretarse 
como procesos y manifestaciones para adaptarse al estrés post-desastre, más que como enfermedades.

A pesar del gran número de investigaciones que evalúan el impacto en la salud mental de las personas después de un desastre natural, la investigación relacionada con los efectos de un terremoto y un tsunami en conjunto es muy escasa, por no decir inexistente. Esto puede deberse a la particularidad del fenómeno en sí y a las dificultades que surgen al momento de evaluar los efectos de ambos desastres naturales en situaciones de emergencia. Sin embargo, dada la intensidad de estos dos eventos, es posible suponer que el efecto en las personas no es el mismo cuando sufren un terremoto por sí solo que cuando sufren una combinación de terremoto y tsunami; esto debiera estar relacionado directamente también con el número de síntomas en cada uno de los trastornos antes mencionados. Por ejemplo, Bödvarsdóttir y Elklit (2004) demostraron la presencia de síntomas de TEPT por el efecto de vivenciar un terremoto en un área afectada en comparación a adultos de un área no afectada. Así mismo, Goenjian, Steinberg, Najarian, Fairbanks, Tashjian y Pynoos (2000), en el terremoto de Spitak, Armenia de 1988, encontraron que personas expuestas a zonas más cercanas al epicentro puntuaron más alto en TEPT, ansiedad y depresión que aquellas que estaban más lejos.

Ahora, cabe preguntarse qué ocurre con otros trastornos que teóricamente están relacionados con los efectos de los desastres naturales, como por ejemplo la CAPA, en el contexto de terremoto y tsunami que aquí se analiza. Al respecto, la literatura es escasa. A pesar de esto, se ha demostrado la relación entre CAPA y la ansiedad (Taylor, 1992); además, existen estudios que prueban que dicha relación es particularmente mayor en etapas específicas del desarrollo como la niñez y la adolescencia (Hayward, Killen, Kraemer y Taylor, 2000; Kearney, Albano, Eisen, Allan y Barlow, 1997; Lau, Calamari y Waraczynski, 1996) al igual que se ha demostrado para las otras variables mencionadas antes. Junto a esto, se sabe que las víctimas de desastres presentan CAPA (Kar, 2009), incluso hasta tres años después de una catástrofe (Önder, Tural, Aker, Kilic y Erdoğan, 2006).

Por consiguiente, dada la particularidad del evento combinado terremoto y tsunami del 27 de febrero en Chile, el presente estudio realizó una primera medición durante las 2 semanas siguientes a la catástrofe en pos de identificar cuáles son las variables relevantes de investigar después de una catástrofe y sus posible relación con la incidencia de ataques de pánico en la población. Para ello se aplicó un cuestionario que recopiló antecedentes demográficos, pérdidas personales y síntomas de CAPA en un grupo de 150 personas pertenecientes a distintas ciudades de la zona centro sur de Chile.

\section{Método}

\section{Participantes}

La muestra estuvo compuesta por 150 adultos (124 hombres y 26 mujeres), todos trabajadores de una gran empresa de distribución con sedes en distintas ciudades, que al momento de la evaluación vivían en las regiones de Valparaíso, Metropolitana, de O'Higgins, del Maule, del Bío-Bío y de la Araucanía. De ellos: 122 viven en ciudades o pueblos del interior $(81,3 \%)$, mientras que 28 lo hacen en lugares cercanos a la costa $(18,7 \%) ; 123$ estaban en sus casas al momento del terremoto $(82 \%)$, mientras que 27 estaban fuera de éstas (18\%). Un total de 78 personas señalaron no haber sufrido ningún daño importante en su vivienda (52\%), 28 haber sufrido daños leves, como grietas $(18,7 \%), 39$ haber sufrido caída de muros (26\%) y 5 haber perdido completamente su casa-habitación (3,3\%). Con respecto al daño en las personas, 141 no han tenido familiares heridos, desaparecidos o fallecidos (94\%), mientras que 9 sí declaran haber tenido familiares afectados físicamente por la catástrofe $(6 \%)$.

\section{Procedimiento e instrumentos}

Para la medición de los aspectos demográficos se utilizó una encuesta que registró las variables sexo, edad, dónde se encontraba durante el terremoto ("en casa" o "fuera de casa"), el lugar de residencia ("costa" o "interior"), el estado de los familiares de cada persona ("sin familiar afectado" o "con familiar afectado"), el grado de daño en su propia vivienda ("sin daños", "grietas", "caída de muros", "pérdida total") y la pérdida de enseres durante la catástrofe ("si" o "no").

Respecto de la medición de los síntomas de CAPA, se confeccionó una lista de chequeo a partir de los 12 síntomas señalados en el DSM-IV (López-Ibor y Valdés, 2008), de cuya suma se obtiene el índice general (CAPA-G). De éstos, 8 corresponden a sensaciones físicas (palpitaciones cardíacas frecuentes, sudoración, sensación de ahogo o falta de aliento, sensación de atragantarse, opresión de malestar cardíaco o en el pecho, náuseas o malestar abdominal, parestesias y escalofríos), mientras que 4 son síntomas de carácter psicológico (desrealización, despersonalización, miedo a perder el control o volverse loco y miedo a la muerte). Del primer y segundo conjunto se obtienen los índices físico (CAPA-F) y psicológico (CAPA-P) del CAPA, respectivamente.

La recolección de datos se realizó entre el 03 y el 15 de marzo de 2010. Los cuestionarios fueron aplicados por personal que estaba en contacto con las personas afectadas. Cada cuestionario fue contestado de manera anónima. 


\section{Plan de análisis}

Se realizaron análisis de la varianza (ANOVA) de un factor por cada variable categórica y para cada una de las variables dependientes CAPA-G, CAPA-F y CAPA-P.

\section{Resultados}

La Figura 1 muestra la media de cada índice para cada una de las variables evaluadas. A modo general, en ella se puede apreciar que no existen diferencias en los índices de CAPA para las variables sexo, dónde se encontraba al momento del terremoto, estado de los familiares y grado de daño de la vivienda ( $p$ s $>.050$ ). Pero sí existen diferencias en las variables "pérdida de enseres" y "ubicación".

Respecto al efecto del factor "ubicación”, el gráfico inferior izquierdo de la Figura 1 muestra que las personas que se encontraban en la costa presentan un nivel más alto de los tres índices de CAPA. Como al realizar la prueba de Levene se determina que no se cumple con el supuesto de homogeneidad de las varianzas de los grupos ( $p s<.001)$, se interpretan los estadísticos robustos de Brown-Forsythe y Welch del ANOVA. Este análisis corrobora estas tendencias, encontrándose un efecto significativo en todos los índices de CAPA (CAPA-G: F $(1,27.086)=15.303 ; \mathrm{p}<0.001 ;$

Figura 1. Media para cada índice de CAPA de las variables: "género" (gráfico superior izquierdo), "dónde se encontraba" (gráfico superior derecho), "grado de daño en su propia vivienda" (gráfico medio izquierdo), "estado de los familiares" (gráfico medio derecho), "lugar de residencia" (gráfico inferior izquierdo) y "pérdida de enseres" (gráfico inferior derecho). Las barras de error representan el error estándar de la media.

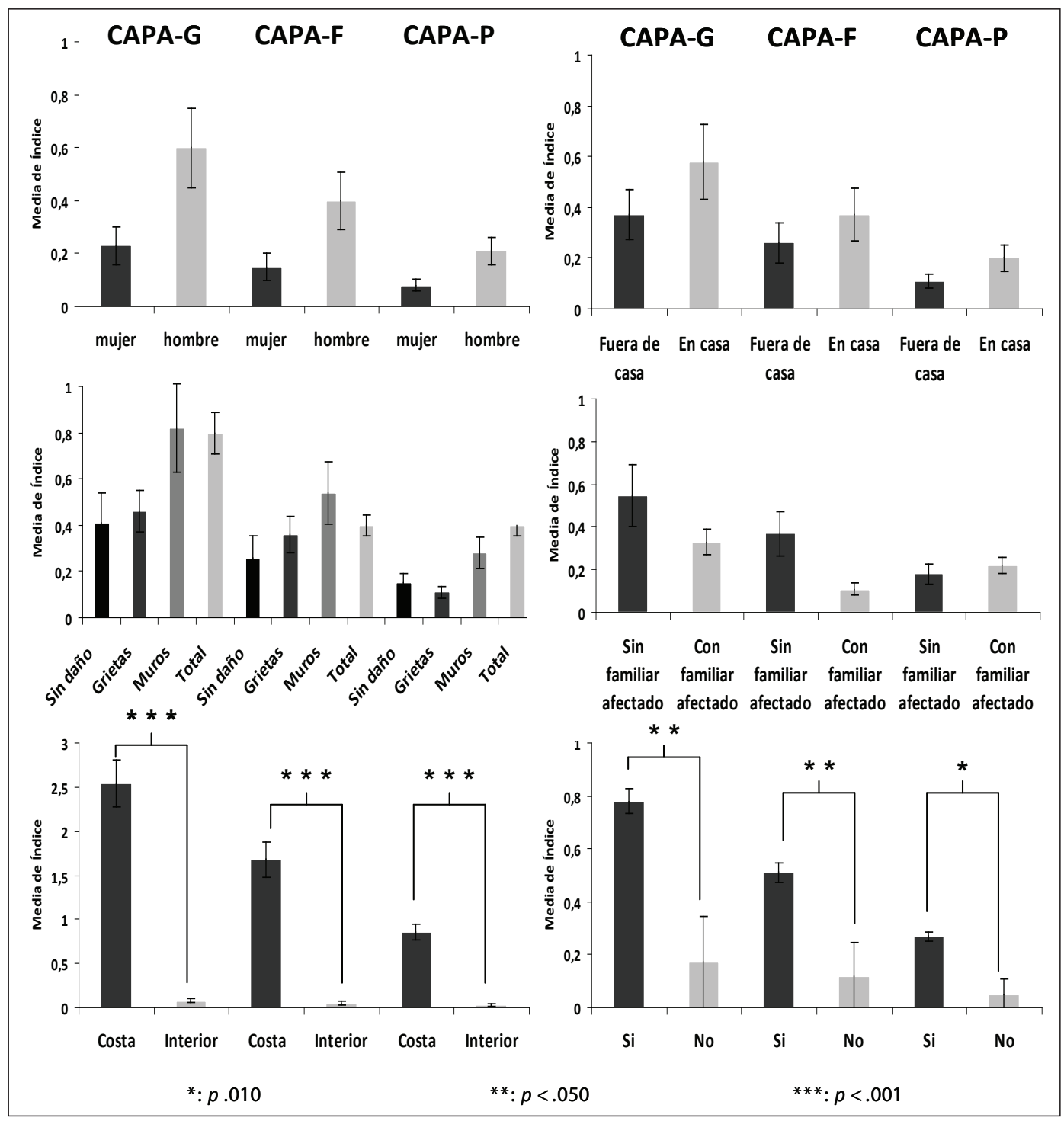


CAPA-F: $\mathrm{F}(1,27.099)=12.513 ; \mathrm{p}<0.001$; CAPA-P: $\mathrm{F}(1$, $27.321)=15.278 ; \mathrm{p}<0.001)$.

Respecto de la variable "pérdida de enseres", el gráfico inferior derecho de la Figura 1 muestra que quienes perdieron enseres presentan un nivel más alto de los tres índices de CAPA que quienes no tuvieron pérdidas En este caso, tampoco se cumple con el supuesto de homogeneidad de las varianzas de los grupos (ps $<0.001)$, interpretándose los estadísticos robustos de Brown-Forsythe y Welch para el ANOVA. El análisis muestra un efecto significativo de todos los índices de CAPA (CAPA-G: F $(1,110.215)=6.667$; $\mathrm{p}<$ 0.050; CAPA-F: $\mathrm{F}(1,113.440)=5.153 ; \mathrm{p}<0.050$; CAPA-P: $\mathrm{F}(1,113.752)=7.471 ; \mathrm{p}<0.010)$.

\section{Discusión}

El 27 de Febrero de 2010, uno de los terremotos más intensos de la historia afectó la zona central de Chile, el cual provocó un tsunami que terminó por devastar las ciudades y localidades costeras. Se espera que ambos eventos catastróficos hayan tenido un efecto concreto no sólo en el medio ambiente físico y social de las personas, sino que también en sus cogniciones, emociones y comportamientos. Los hallazgos de este estudio proporcionan una primera confirmación de esto. Quienes perdieron enseres presentan más síntomas de CAPA que quienes no perdieron enseres; y quienes viven en la costa presentan más síntomas que quienes viven en el interior. Esto corrobora hallazgos anteriores sobre el efecto de la pérdida de los bienes materiales en la prevalencia de síntomas de distintos trastornos psiquiátricos (Shara, Chaudhary, Kavathekar y Saxena, 1996). Lo anterior podría explicarse dado que el grado de destrucción ambiental de un terremoto se vería amplificado con un tsunami, lo cual tendría un efecto potenciador en las respuestas conductuales, cognitivas y emocionales en aquellas personas que vivenciaron ambos eventos, como es el caso de aquellas del sector costero del litoral central.

Desde los modelos causales clásicos de la conducta social, el comportamiento de la persona se concibe en función de los eventos de su ambiente pudiendo reevaluar su conducta a partir de las consecuencias de la misma en función del tiempo (Kanfer y Phillips, 1980). Tomando en consideración lo anterior y los parámetros de impacto de cualquier evento traumático señalados por la OPS (2006), podemos establecer que las personas del sector costero manifiestan más síntomas de pánico y ansiedad porque han estado expuestas a un tsunami después de haber vivido un terremoto. Ambos eventos conjuntamente poseen un mayor poder destructivo, que se traduce en mayores pérdidas materiales y familiares, y en un mayor número de consecuencias psicológicas, como el aumento de los síntomas vinculados con los trastornos de ansiedad. Sin embargo, este tipo de modelos ha evolucionado a otros más complejos en donde se plantea una reciprocidad entre el ambiente, la conducta y factores internos o personales (Bandura, 1974). Aquí, la información confluye desde distintas fuentes activando mecanismos regulatorios de auto observación en las personas pudiendo actuar de forma diferencial a los mismos estímulos en situaciones distintas. Bajo este enfoque, los efectos en la salud mental de las personas se ven mayormente alterados por la sucesión del tsunami posterior al terremoto, esperándose un mayor impacto en estas personas. No obstante, la respuesta también está mediada por otro tipo de variables personales y sociales. Por ejemplo, en el estudio del impacto en la salud mental de personas expuestas a catástrofes, se ha demostrado la asociación del TEPT, la ansiedad y la depresión con variables personales tales como el estilo de afrontamiento (Carr, Lewin, Kenardy, Webster, Hazell, Carter y Williamson, 1997) y componentes hereditarios (Goenjian, Noble, Walling, Goenjian, Karayanc, Ritchieb y Bailey, 2008), y también sociales como el soporte percibido, la sobreprotección y la forma de resolver conflictos parentales (Bokszczanin, 2008).

Desde un punto de vista aplicado, los hallazgos de esta investigación demuestran preliminarmente que la atención en salud mental asistencial debe ser focalizada. Esto se puede lograr más eficientemente a partir de un catastro que indique los daños materiales (pérdida de enseres), que incorpore variables de localización geográfica de las personas al momento de la catástrofe. Respaldan esto otros estudios que comparan la intensidad de exposición entre personas cercanas o lejanas al epicentro de un terremoto (Goenjian, Steinberg, Najarian, Fairbanks, Tashjian y Pynoos, 2000). De esta manera, los planes de contingencia post-terremoto pueden ejecutarse de manera más eficiente y acotada.

Por otro lado, la presencia de este tipo de síntomas se ha encontrado incluso hasta años después de la catástrofe (Jensen, Dyb y Nygaard, 2009). Por consiguiente, se hace imprescindible la realización de estudios longitudinales que permitan dar cuenta del progreso y estado futuro de las personas afectadas, los procesos de recuperación, el impacto de las políticas de salud mental, la efectividad de los tratamientos y el impacto sobre aquellos grupos vulnerables. Y, respecto de las intervenciones para trabajar con las víctimas de catástrofes, es altamente importante comprobar su efectividad. En ese sentido, se recomienda realizar las intervenciones según el diseño básico de al menos dos grupos de personas (para comparar lo que ocurre con un grupo en relación con otro) y por lo menos dos mediciones de las variables que se están evaluando (una antes y otra después de la intervención).

Es pertinente mencionar también que existen ciertas limitaciones de este estudio que tienen que ver con el tamaño de los grupos y con el cumplimiento de los supuestos para todas las comparaciones (normalidad de la distribución y homogeneidad de varianzas), lo cual podría explicar la ausencia de diferencias por género, cuando se ha determinado en estudios anteriores a las mujeres como 
una población de riesgo. La necesidad de evaluar rápidamente a las personas afectadas y las dificultades propias ante una situación de catástrofe, redundaron en la falta de rigor en este aspecto. Aun así, los resultados proveen una evaluación preliminar de los efectos provocados por esta catástrofe que coincide con hallazgos anteriores y pueden guiar futuras investigaciones.

Estudios posteriores podrán complementar estos datos incluyendo algún tipo de indicador cuantitativo del nivel de impacto objetivo sufrido por las personas, el cual se podría construir como un indicador compuesto que considere la magnitud del terremoto sufrido en la zona de residencia, el grado de destrucción de la vivienda, el impacto económico individual, pérdida de vidas y daño físico, entre otros. Un indicador de este tipo permitirá estudiar con mayor rigor y riqueza las alternaciones psicológicas que se desprenden de este tipo de catástrofes.

Finalmente, a la luz de los resultados, de la investigación psicosocial en este tipo de fenómenos y de las consecuencias de esta catástrofe, consideramos muy necesario que los equipos de emergencia conozcan los factores psicosociales involucrados en una catástrofe, así como que la incorporación de psicólogos y psicólogas a estos equipos para el soporte, recuperación y rehabilitación de la población. Esto, tanto al momento de la catástrofe y el proceso de reconstrucción, como en el estudio y creación de políticas de prevención y acción para evitar al máximo las pérdidas humanas y mitigar el daño de los afectados (ver Atwater, Cisternas, Bourgeois, Dudley, Hendley II y Stauffer, 2005; Chan, Gao y Griffiths, 2009; Rao, 2006). Así, es necesario realizar más y mejor investigación que permita dar nuevos y mejores lineamientos para la prevención y la intervención en el caso de nuevos desastres naturales. La historia de este tipo de desastres en nuestro país justifica con creces tal esfuerzo.

\section{Referencias}

Atwater, B.F., Cisternas, M.V., Bourgeois, J., Dudley, W.C., Hendley, J.W., \& Stauffer, P.H. (2005). Surviving a Tsunami - Lessons from Chile, Hawaii, and Japan. Circular 1187, v 1.1, Disponible: http:// pubs.usgs.gov/circ/c1187/

Bandura, A. (1974). Behavior theory and models of man. American Psychologist, 29, 859-869.

Bokszczanin, A. (2008). Parental support, family conflict, and overprotectiveness: Predicting PTSD symptom levels of adolescents 28 months after a natural disaster. Anxiety, Stress, \& Coping, 21, 325-335.

Bödvarsdóttir, I., \& Elklit, A. (2004). Psychological reactions in Icelandic earthquake survivors. Scandinavian Journal of Psychology, 45, 3-13.

Cairo, J.B., Dutta, S., \& Nawaz, H. (2010). The prevalence of posttraumatic stress disorder among adult earthquake survivors in Peru. Disaster Medicine and Public Health Preparedness, 4, 39-46.

Carr, V.J., Lewin, T.J., Kenardy, J.A., Webster, R.A., Hazell, P.L., Carter, G.L., \& Williamson, M. (1997). Psychosocial sequelae of the 1989 Newcastle earthquake: III. Role of vulnerability factors in postdisaster morbidity. Psychological Medicine, 27, 179-190.

Chan, E.Y.Y., Gao, Y., \& Griffiths, S.M. (2009). Literature review of health impact post-earthquakes in China 1906-2007. Journal of Public Health, 32, 52-61.
Cohen, R. (2008). Lecciones aprendidas durante desastres naturales. Revista Peruana de Medicina Experimental y Salud Pública, 25, 109-117. Goenjian, A.K., Noble, E.P., Walling, D.P., Goenjian, A.G., Karayanc, I.S., Ritchieb, T., \& Bailey, J.N. (2008). Heritabilities of symptoms of posttraumatic stress disorder, anxiety, and depression in earthquake exposed Armenian families. Psychiatric Genetics, 18, 261-266.

Goenjian, A.K., Steinberg, A.M., Najarian, L.M., Fairbanks, L.A., Tashjian, M., \& Pynoos, R.S. (2000). Prospective study of posttraumatic stress, anxiety, and depressive reactions after earthquake and political violence. American Journal of Psychiatry, 157, 911-916.

Groome, D., \& Soureti, A. (2004). Post-traumatic stress disorder and anxiety symptoms in children exposed to the 1999 Greek earthquake. British Journal of Psychology 95, 387-397.

Harville, E.W., Xiong, X., Buekens, P., Elkind-Hirsch, K., \& Pridjian, G. (2009). Post-traumatic stress disorder, depression, and resilience after hurricane Gustav. American Journal of Epidemiology, 169(Suppl): S1-S137.

Hayward, C.W., Killen, J.D., Kraemer, H.C., \& Taylor, C.B. (2000) Predictors of panic attacks in children and adolescents. Journal of the American Academy of Child and Adolescent Psychiatry, 39, 207-14.

INE-Instituto Nacional de Estadísticas (2003). Censo 2002: Síntesis de Resultados. Santiago: La Nación, pp. 9, Disponible: www.ine.cl/cd2002/ sintesiscensal.pdf

Jensen, T., Dyb, G., \& Nygaard, E. (2009). A longitudinal study of posttraumatic stress reactions in norwegian children and adolescents exposed to the 2004 tsunami. Archives of Pediatric Adolescent Medicine, 163, 856-861.

Kanfer, R., \& Phillips, J. (1980). Principios de Aprendizaje en la Terapia del Comportamiento. México: Trillas.

Kar, N. (2009). Psychological impact of disasters on children: review of assessment and interventions. World Journal of Pediatrics, 5, 5-11.

Kar, N., Mohapatra, P.K., Nayak, K.C., Pattanaik, P., Swain, S.P., \& Kar, H.C. (2007). Post-traumatic stress disorder in children and adolescents one year after a super-cyclone in Orissa, India: exploring cross-cultural validity and vulnerability factors. BMC Psychiatry, 7, 1-9.

Kearney, C.A., Albano, A.M., Eisen, A.R., Allan, W.D., \& Barlow, D.H. (1997). The phenomenology of panic disorder in youngsters: an empirical study of a clinical sample. Journal of Anxiety Disorders, 11, 49-62.

Khan, M., Masood, M., Mukhtar, M., Sana, N., \& Chaudhry, H. (2010). Gender differences in prevalence of anxiety disorders among earth quake survivors. 18th European Congress of Psychiatry, 25, 351.

Lau, J.J., Calamari, J.E., \& Waraczynski, M. (1996). Panic attack symptomatology and anxiety sensitivity in adolescents. Journal of Anxiety Disorders, 10, 355-64.

López-Ibor, J.J., \& Valdés, M. (2008). DSM-IV. Manual diagnostico y estadístico de los trastornos mentales. Madrid: Masson.

McFarlane,A.C., \& Papay, P. (1992). Multiple diagnoses in posttraumatic stress disorder in the victims of a natural disaster. Journal of Nervous and Mental Disease, 180, 498-504.

Ministerio del Interior-Gobierno de Chile (2010a). Fallecidos confirmados con RUT y certificado de defunción de registro civil (actualizado al 15/04/2010). Recuperado en Julio 14, 2010 disponible en http://www. interior.gov.cl/

Ministerio del Interior-Gobierno de Chile (2010b). Denuncias por presunta desgracia relacionadas con el terremoto y maremoto del 27 de febrero (actualizado al 15/04/2010). Recuperado en Julio14, 2010 disponible en http://www.interior.gov.cl/

Organización Panamericana de la Salud (2006). Guía práctica de salud mental en situaciones de desastres. Washington D.C.: paho.org.

Önder, E., Tural, Ü., Aker, T., Kılıc, C., \& Erdoğan, S. (2006). Prevalence of psychiatric disorders three years after the 1999 earthquake in Turkey: Marmara Earthquake Survey (MES). Social Psychiatry and Psychiatric Epidemiology, 41, 868-874.

Priebe, S., Grappasonni, I., Mari, M., Dewey, M., Petrelli, F., \& Costa, A. (2009). Posttraumatic stress disorder six months after an earthquake. Social Psychiatry and Psychiatric Epidemiology, 44, 393-397.

Rao, K. (2006). Lessons learnt in mental health and psychosocial care in India after disasters. International Review of Psychiatry, 18, 547-552. 
Riquelme, P. (2010, febrero 28). Tres enormes olas devastan Constitución tras sismo. La Tercera, p.8.

Riquelme, C. (2010). El terremoto en Chile deja 795 muertos y dos millones de damnificados. Europapress [En línea] Ed.03 de marzo de 2010. Disponible: http://www.europapress.es/internacional/noticia-terremotochile-deja-795-muertos-dos-millones-damnificados-20100302225048. $\mathrm{html}$

Rochanakorn, K. (2007). IASC Guidelines on Mental Health and Psychosocial support in Emergency settings [En línea], Disponible: http://www. who.int/hac/network/interagency/news/mental_health_guidelines/en/

Sharan, P., Chaudhary, G., Kavathekar, S., \& Saxena, S. (1996). Preliminary report of psychiatric disorders in survivors of a severe earthquake. American Journal of Psychiatry, 153, 556-558.

Solvason, H.B., Ernst, H., \& Roth, W. (2003). Predictors of response in anxiety disorders. Psychiatric Clinics of North America, 26, 411-433.
Taylor, S. (1992). How does anxiety sensitivity vary across the anxiety disorders? Journal of Anxiety Disorder, 6, 249-259.

Thienkrua, W., Lopes Cardozo, B., Somchai Chakkraband, M.L., Guadamuz, T.E., Pengjuntr, W., Tantipiwatanaskul, P., Sakornsatian, S., Ekassawin, S., Panyayong, B., Varangrat, A., Tappero, J.W., Schreiber, M., \& van Griensven, F. (2006). Symptoms of posttraumatic stress disorder and depression among children in tsunami-affected areas in southern thailand. Journal of the American Medical Association, 296, 549-559.

Ticehurst, S., Webster, R.A., Carr, V.J., \& Lewin, T.J. (1996). The psychosocial impact of an earthquake on the elderly. International Journal of Geriatric Psychiatry, 11, 943-951.

USGS - United States Geological Survey (2010). Tsunami and earthquake research. Recuperado en Julio 14, 2010 disponible en http://walrus. wr.usgs.gov/tsunami/ 
